\title{
Practical Single MPP Absorber
}

\author{
Dah-You Maa ${ }^{\dagger}$ \\ Institute of Acoustics CAS, POB 2712, Beijing 100080, China
}

(Received 8 September 2006; accepted 15 January 2007)

\begin{abstract}
The construction and properties of microperforated panel (MPP) absorbers are discussed. MPP is a plate perforated with numerous sub-millimeter orifices forming a resonant sound absorber with a cavity behind. It has been shown that low values of the perforate constant $k$ and the orifice diameter $d$ are essential for the MPP to have high absorption over a wide frequency band. To find the exact absorption limits, one can assume for that $k=1$ as a start, because both specific resistance and wide frequency bands of absorption require $k$ to be one or less. The orifice diameter $d$ is chosen as $0.1 \mathrm{~mm}$, so that the peak absorption coefficient (resonance absorption) is at $1000 \mathrm{~Hz}$, and the higher sound frequencies may be included in the absorption region. Is it possible for a single MPP to cover the whole absorption region required in practice? The half-absorption limit and 0.5 absorption coefficient limit were used as criteria for comparison with different load resistances. MPP absorbers designed for use typically absorb sound over approximately two octaves, and the new absorbers with low values of $k$ and $d$ are found to be better for $r=1$ (relative acoustical resistance equal to the characteristic impedance $\rho c$ in air), over about 2.5 octaves. This increases to around 3 octaves for $r=2$ or 3 . In addition, the MPP in a reverberant sound field will absorb over a wider frequency ranges due to increased high frequency absorption. If the increase in range is enough, the absorption region will join forces with the secondary absorption regions, due to the multi-resonance property of the resonance structure, to form a continuous absorption region. The design of a single MPP absorber is given and its realisation is discussed.
\end{abstract}

${ }^{\dagger}$ Honorary Fellow of the International Institute of Acoustics and Vibration (IIAV)

\section{INTRODUCTION}

A microperforated panel (MPP) absorber is made of thin sheet metal drilled or punched with tens of thousands of minute orifices of sub-millimetre diameter with a cavity behind and is used to absorb sound without using any porous, fibrous absorbing materials. Such absorbers have been found to be good, and better than any other resonant absorber, but the frequency range is not quite enough for a practical applications. Further development has been made to extend the absorption frequency range by using a double resonator; two similar MPP sheets with individual cavities in tandem are used. The results were satisfactory. Theory was developed in $1975,{ }^{1}$ and simplified later, ${ }^{2}$ to allow MPP absorber properties to be precisely predicted. Both structure and absorption characteristics can be exactly predicted from given values of the relative load resistance $r$ and perforate constant $k=d(\omega \rho / 4 \eta)^{1 / 2}$, where $d$ is the diameter of orifice, and the resonant angular frequency is $\omega=2 \pi f$. MPP absorbers have sufficient acoustic resistance in themselves and low mass reactance suitable for a wide frequency band absorber without any fibrous and porous absorbing material. The panels can be made with any material suitable for the application and environment, and are easy to prepare and to design exactly according to the requirements. It has been applied successfully ever since with important progress of the technology and public interest. ${ }^{3}$

Is it possible to design a practical single MPP absorber to cover the whole frequency range of interest in noise control? A rough estimate has been made ${ }^{4}$ that this is possible provided low values of $k$ and $d$ are used. The purpose of the present investigation is to find whether the estimation can be realised by taking $k=1$, and $d=0.1 \mathrm{~mm}$ as a start and to find the exact requirements for a practical single MPP absorber. Realisation of the single MPP absorber is considered.

\section{THE MPP ABSORBER ${ }^{1}$}

The MPP absorber and its equivalent circuit are shown in Fig. 1. The absorption coefficient of the MPP absorber at normal incidence is

$$
a=\frac{4 r}{(1+r)^{2}+[\omega m-\cot (\omega D / c)]^{2}},
$$

where the relative impedance $r+j \omega m$ of the MPP is the ratio of the specific acoustic impedance per unit area $Z=R+j \omega M$ divided by the characteristic impedance $\rho c$ in air ( $\rho$ being the density and $c$ the sound velocity in air), and may be found as

$$
\begin{aligned}
& r=\frac{32 \eta}{\sigma \rho c} \frac{t}{d^{2}} k_{r} ; \quad k_{r}=\left(1+\frac{k^{2}}{32}\right)^{1 / 2}+\frac{\sqrt{2}}{32} \frac{k d}{t} \\
& \omega m=\frac{\omega t}{\sigma c} k_{m} ; \quad k_{m}=1+\left(9+\frac{k^{2}}{2}\right)^{-1 / 2}+0.85 \frac{d}{t},
\end{aligned}
$$

or

$$
\omega m=\frac{1}{8} \frac{k_{m}}{k_{r}} r k^{2}
$$

and

$$
k=d \sqrt{\omega \rho / \eta}=10 d \sqrt{f},
$$

as the value of viscosity is substituted.

The constants are: the thickness of the panel $t$, the diameter of the orifice $d$, the sound frequency $f$, the depth of the cavity behind the panel $D$, the coefficient of viscosity $\eta$ in air, $1.8 \times 10^{-5} \mathrm{~kg} / \mathrm{ms}$, and the porosity $\sigma$ on the panel, all in SI units.

The maximum value of the absorption coefficient is given by

$$
a_{0}=\frac{4 r}{(1+r)^{2}}
$$

